\title{
Seminal Levels of Pro-inflammatory (CXCL1, CXCL9, CXCL10) and Homeostatic (CXCL12) Chemokines in Men With Asymptomatic Chlamydia trachomatis Infection
}

\author{
Hamid Hakimi ${ }^{1}$; Nahid Zainodini ${ }^{1}$; Hossein Khorramdelazad ${ }^{2}$; Mohammad Kazemi \\ Arababadi ${ }^{1}$; Gholamhossein Hassanshahi ${ }^{2,}$ \\ ${ }_{1}^{1}$ Immunology of Infectious Diseases Research Center, Rafsanjan University of Medical Sciences, Rafsanjan, IR Iran \\ ${ }_{2}^{2}$ Molecular Medicine Research Center, Rafsanjan University of Medical Sciences, Rafsanjan, IR Iran \\ ${ }^{*}$ Corresponding author: Gholamhossein Hassanshahi, Molecular Medicine Research Center, Rafsanjan University of Medical Sciences, Rafsanjan, IR Iran. Tel: +98-3915234003-5/+98- \\ 9133933447, Fax: +98-3915225209, E-mail: ghhassanshahi@rums.ac.ir
}

Recived: April 09, 2013; Revised: July 17, 2013; Accepted: July 20, 2013

\begin{abstract}
Background: Chemokines play important roles in immune system activation against microbial infections.
objectives: The current study aimed to evaluate seminal levels of CXC chemokines CXCL1, CXCL9, CXCL10 and CXCL12 in Chlamydia trachomatis infected patients.

Materials and Methods: The C. trachomatis infection was determined employing Polymerase Chain Reaction (PCR)-based methods. Seminal concentrations of CXCL1, CXCL9, CXCL10 and CXCL12 were measured by Enzyme-Linked Immunosorbent Assay(ELISA).

Results: The current study results demonstrated that the semen levels of CXCL1 and CXCL9, but not CXCL10 and CXCL12, significantly increased in C. trachomatis infected patients compared to the healthy controls.

Conclusions: Based on the current study results, it may be concluded that both CXCL1 and CXCL9 play more important roles than CXCL10 and CXCL12 in induction of immune responses against C. trachomatis and could possibly be considered as future targets for immunotherapy of $C$. trachomatis infection.
\end{abstract}

Keywords:Chlamydia trachomatis; Chemokine, CXCL1, CXCL9, CXCL10 and CXCL12

\section{Background}

The Chlamydia trachomatis, is an obligate Gram negative intracellular bacterial parasite. It is one of the most prevalent sexually-transmitted pathogens inducing permanent complications in infected subjects, worldwide. The developmental cycle of these bacteria consists of two forms as follow: A. Elementary Body (EB), that is the extracellular infectious form and metabolically inactive; B. Reticulate Body (RB), the intracellular metabolically active form dividing by binary fission. C. trachoma$t i s$ is involved in asymptomatic genital tract infections in both women and men $(1,2)$. C. trachomatis infection causes more severe complications in females than males (1). This infection leads to different diseases varying from acute and chronic pelvic inflammatory diseases, cervicitis, urethritis, endometritis, infertility and ectopic pregnancy (3), to different urogenital infections such as urethritis, prostatitis, and epididymitis (4). To date, the effect of these bacteria on male fertility is yet to be clarified. Several immunological factors including, antibodies (Abs), cytokines and chemokines have been reported to be involved in male infertility by several researchers $(5,6)$. Elevated levels of these factors in semen of men in- fected with $C$. trachomatis offer a role in immune defense of the male genital tract (7).

Previous reports (an ectocervical and endocervical cells, human urethral epithelial cells, and immortalized normal adult male prostate epithelial cells) demonstrated that chemokines play important roles in immune responses against this bacterium $(5,8)$. Chemokines are defined as a homologous group of 8-10 kDa peptides. They are further divided into four various subgroups including $\mathrm{CXC}, \mathrm{CC}, \mathrm{C}$, and $\mathrm{CX} 3 \mathrm{C}$ based on the position of cystein molecules in their biochemical configuration (9). All members of chemokine family, including CXC signal through specific seven transmembrane-G protein coupled receptors (CXC chemokine receptors (CXCRs)) (10).

According to the presence or absence of N-terminus conserved motif identified as ELR (containing glutamate, leucine, arginine) CXC chemokines themselves are further subdivided into two subclasses as ELR+ and ELR-(6, 11). CXCL1 and CXCL12 fit in ELR positive CXC chemokines while CXCL9 and CXCL10 are ELR-negative $(12,13)$. ELR-positive (CXCL12 and CXCL1) and ELR-(CXCL9 and CXCL10) CXC chemokines are neutrophils and $\mathrm{T}$ lymphocytes recruiters,

Copyright ( 2014 ,Ahvaz Jundishapur University of Medical Sciences; Published by Kowsar. This is an open-access article distributed under the terms of the Creative Commons Attribution-NonCommercial 4.0 International License (http://creativecommons.org/licenses/by-nc/4.0/) which permits copy and redistribute the material just in noncommercial usages, provided the original work is properly cited. 
respectively. Therefore, increased expressions of these chemokines in response to C. trachomatis result in inflammation in the infected tissues. Activation of these chemokines is frequent in autoimmune and inflammatory disorders (14). Accordingly, previous studies demonstrated that immune, including chemokines, responses against $C$. trachomatis determine the outcome of infection (15). Additionally, during infection with C. trachomatis, an inflammation occurs due to the predominant roles of these chemokines in recruitment and activation of lymphocytes.

\section{Objectives}

The current study aimed to evaluate the relationship between the seminal levels of CXCL1, CXCL9, CXCL10 and CXCL12 in patients with C. trachomatis infection.

\section{Materials and Methods}

\subsection{Collection of Semen Samples}

During this cross-sectional study, 585 consecutive men attending at the Avesina Research Institute, Tehran-Iran, were selected for the study. Ethical Committee for Researches in Rafsanjan University of Medical Sciences approved using semen samples for the analysis. All of the participants including patients with $C$. trachomatis infection and the healthy controls were free of sexually transmitted diseases and had not received chemotherapy or radiotherapy treatment. None of them had the background of vasectomy, and abnormally low semen volume; a retrograde ejaculation and hypogonadism were considered as excluding criteria. All of the participants underwent semen analysis for infertility investigations. They were asked to avoid sexual intercourse for two to five days before attending the examination. The healthy controls were age/sex matched with the C. trachomatis infected patients. All of the semen samples were collected into standard containers according to the methods outlined in World Health Organization (WHO) laboratory manual (16). Semen samples were stored at $-20^{\circ} \mathrm{C}$ for further studies.

\subsection{DNA Extraction}

A DNA extraction Kit (Bioneer, South Korea) was used for semen samples according to the manufacturer's guidelines. The extracted DNA was checked using $\beta$-actin primers to examine either the presence of PCR inhibitors or DNA quality, then the resultant DNA was stored at $-20^{\circ} \mathrm{C}$ until using in PCR analysis.

\subsection{PCR Condition}

PCR was performed by forward (5'-GGACAAATCGTATCTCGG-3') and reverse (5'-GAAACCAACTCTACGCTG-3') primers (Cinaclon, Iran) specific for K/SotonK1 plasmid pSotonK1 gene of 517 base pair (bp) C. trachomatis to diagnose the infection. The following program was used for PCR amplification: 35 cycles of $93^{\circ} \mathrm{C}$ for 60 seconds, $60^{\circ} \mathrm{C}$ for $20 \mathrm{sec}$ - onds, and $72^{\circ} \mathrm{C}$ for 40 seconds and C. trachomatis genome strain K1 was used as positive control.

\subsection{Chemokine Assays on Semen Samples}

Concentrations of CXCL1, CXCL9, CXCL10 and CXCL12 in semen were measured by ELISA (R\&D Systems, Minneapolis, UK). The sensitivity of kits was $2 \mathrm{pg} / \mathrm{mL}$ and inter and intra-assay assessments of reliability of the kits were conducted and data were only used when inter and intra assays produced scores of $\mathrm{CV}<14 \%$ and $\mathrm{CV}<3 \%$, respectively.

\subsection{Statistical Analysis}

Results of the current article were presented as mean \pm SEM (standard error of mean) for numeric variables, and absolute frequencies and percentages for categorical variables. Student's t-test was used for analysis of continuous variables including CXCL1, CXCL9, CXCL10 and CXCL12 and the normality of the distribution of the data was examined by applying the Kolmogorov-Smirnov test. In order to perform the statistical analysis, SPSS software version 18.0 (SPSS Inc. Chicago, IL) was employed. All P Values were twotailed, with statistical significance defined as $\mathrm{P} \leq 0.05$.

\section{Results}

The current study results indicated that only 50 out of 585 semen samples (8.55\%) were infected with C. trachomatis (based on the PCR results) (Figure 1). The obtained results demonstrated that the mean semen levels of CXCL1 in the patients and controls were $54.13 \pm 3.76 \mathrm{pg} /$ $\mathrm{mL}$ and $39.91 \pm 2.17 \mathrm{pg} / \mathrm{mL}$, respectively $(\mathrm{P}=0.027)$ (Figure 2). It was observed that the seminal CXCL9 levels were $3742.71 \pm 145.75 \mathrm{pg} / \mathrm{mL}$ and $1488 \pm 75.27 \mathrm{pg} / \mathrm{mL}$ in the patients and controls, respectively $(\mathrm{P}<0.001)$ (Figure 3$)$. The CXCL10 levels were $403.18 \mathrm{pg} / \mathrm{mL} \pm 9.57 \mathrm{pg} / \mathrm{mL}$ and 395.67 $\pm 8.02 \mathrm{pg} / \mathrm{mL}$ in the patients and controls, respectively ( $\mathrm{P}$ $=0.729$ ). Finally it was also found that the seminal CXCL12 levels were $35.07 \pm 1.73 \mathrm{pg} / \mathrm{mL}$ and $41.21 \pm 7.96 \mathrm{pg} / \mathrm{mL}$ in the patients and controls, respectively $(\mathrm{P}=0.470$ ) (Figure 4).

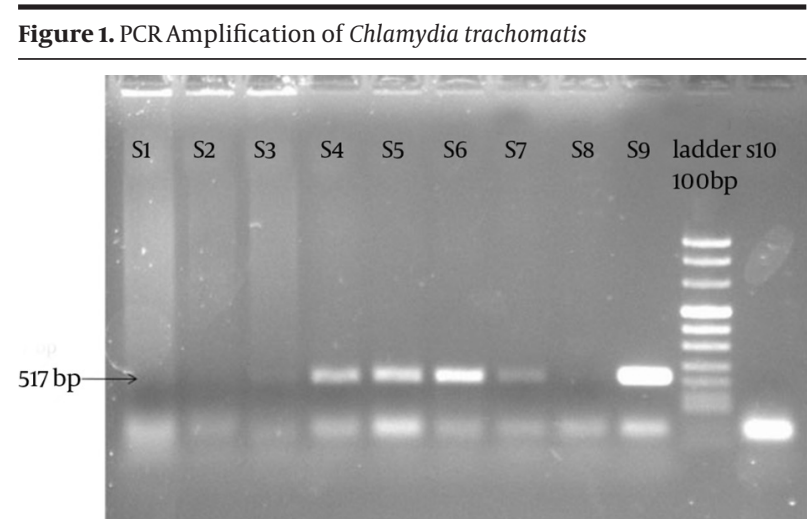

The figure presents PCR amplification of C. trachomatis K/SotonK1 plasmid pSotonK1 (517 bp). Sample 1 (S1), S2 and S3 as well as S8 presents the negative samples and negative control, respectively. S4, S5 and S6 are positive samples and S9 is positive control. 


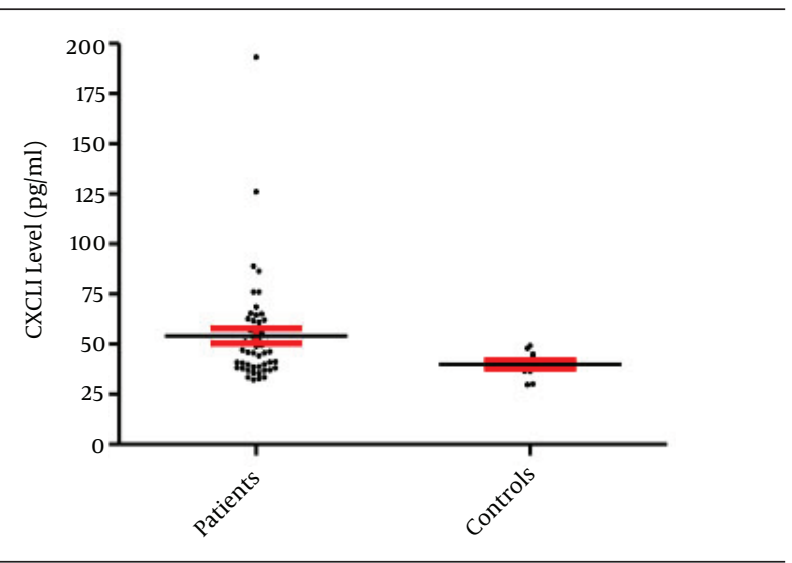

Figure 2. Seminal Levels of CXCL1 in Men with Asymptomatic Chlamydia trachomatis Infection and Control

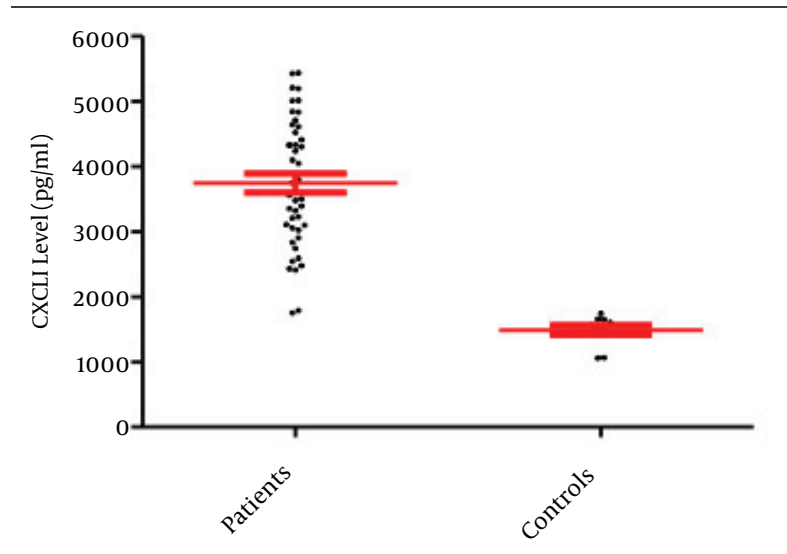

Figure 3. Seminal Levels of CXCL9 in Men with Asymptomatic Chlamydia trachomatis Infection and Control

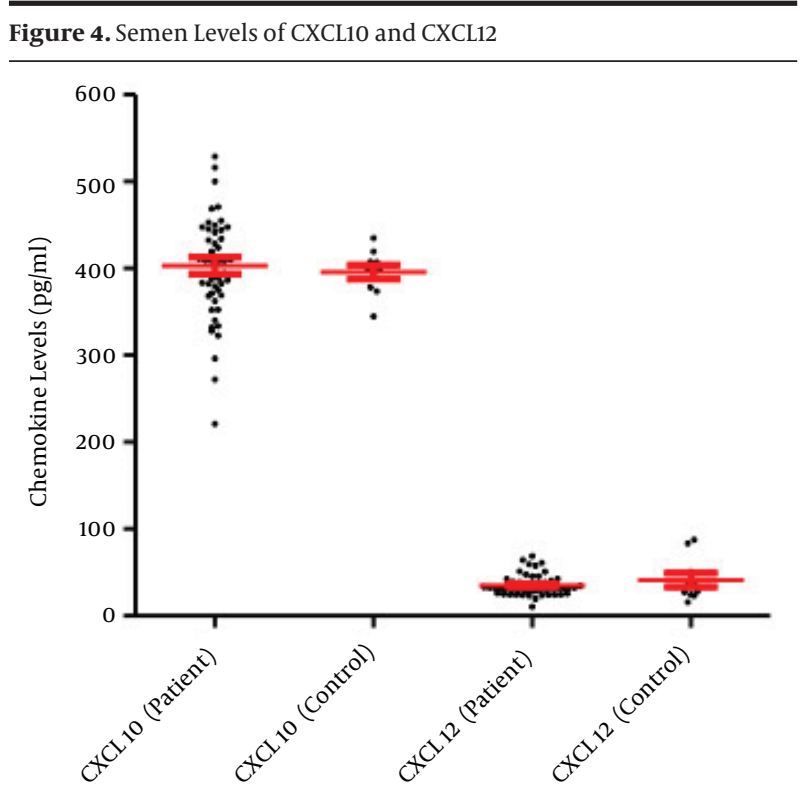

The figure illustrates no significant increase of the semen levels of CXCL10 and CXCL12, patients infected with Chlamydia trachomatis, and the controls.
Overall, results of the present investigation revealed that the levels of CXCL1 and CXCL9 were significantly different from those of the controls and there was a positive correlation between chemokines and infection. It was also observed that although CXCL10 followed the pattern of these inflammatory (CXCL1 and CXCL9) chemokines, its elevated level was not significant. Ultimately, sustained seminal levels of CXCL12 in patients and controls were observed.

\section{Discussion}

In the present study, the semen levels of CXCL1, CXCL9, CXCL10 and CXCL12 were investigated in patients with $C$. trachomatis infection and healthy controls. Notably, semen levels of both CXCL1 and CXCL9 significantly increased in patients, which may be possibly related to their functions as pro-inflammatory. Chemokines are involved in the processes of neutrophil and monocyte lineage infiltration into the infected testis. It is well established that CXCL1 acts as migratory factor for neutrophils while CXCL9 is a lymphocyterecruiter. It has also been well evidenced that the innate immune responses of epithelial cells and urogenital tract resident immune cells are the first line of defense against $C$. trachomatis (17). Furthermore, chemokines and cytokines are produced during the innate immune responses of the chemoattract inflammatory cells against the infected tissues to control infection.

Chemokines also initiate adaptive immune response against the bacteria and further clearance processes (18). Therefore, based on the current study results, it may be concluded that both CXCL1 and CXCL9 are critical chemokines involved in the innate immune responses against C. trachomatis infection. Previous studies demonstrated that CXCL1 is produced by several immune and nonimmune tissue/cell systems, including macrophages, neutrophils and epithelial cells with predominant neutrophil chemoattraction activities (19). Moreover, it has also been documented that CXCL9 play important roles in infiltrating of the activated $\mathrm{T}$ lymphocytes towards the infected and inflamed tissues (20). Thus, CXCL1 and CXCL9 may regulate activation and infiltration of neutrophils and T lymphocytes to the infected regions allowing progression of immune responses against C. trachomatis. Although, several research teams reported the presence of pro-inflammatory cytokines in the semen of patients infected with C.trachomatis, to best of the authors' knowledge, the present study is the first to address elevated levels of CXCL1 and CXCL9 in semen of these patients. The other member of IFN- $\gamma$ inducible inflammatory chemokines (CXCL10) also increased, which was not significant. Elevated levels of these chemokines as downstream targets of IFN- $\gamma$ could be presumably related to their function as members of inflammation pathway rather than their other properties.

A previous study demonstrated that the expression of CXCL9 depends on IFN- $\gamma$ and activation of its signaling pathway transcription factors, especially STAT1 (21). Additionally, the ability of the male urogenital tract epithe- 
lial cells to produce IFN- $\gamma$ has been documented and this cytokine is present in seminal fluid (22). Studies revealed that both CXCL1 and CXCL9 are inducible by IFN- $\gamma$ and other pro-inflammatory cytokines such as TNF- $\alpha$ (13). It suggests that production of IFN- $\gamma$ in response to bacterial infections, including $C$. trachomatis, may induce the expression of CXCL9. In agreement with the results of the current study, several other research teams reported that subsequent to infection with $C$. trachomatis, epithelial cells produce a wide variety of pro-inflammatory mediators, including CXCL1, CXCL8, CXCL16, granulocyte/ monocyte-colony stimulating factor (GM-CSF), IL-1, IL-6, and TNF $\alpha$ (23). Mackern Oberti et al. have examined the prostate epithelial/stromal cells dependent immune responses to $C$. trachomatis and observed that it could infect murine prostate cells by the development of large inclusions. Moreover, their results demonstrated that prostate cells have responded to $C$. trachomatis infection by production of pro-inflammatory chemokines inducing CCL2, CXCL1, and CXCL2 (19). They also observed that the chemokine productions by prostate cells were performed via activation with toll-like receptor, pathogen-associated molecular patterns interaction (19).

Finally, according to the findings of the current study and the other aforementioned studies, it may be concluded that several men genital system cell types such as prostate, epithelial and resident immune cells are involved in the development of immunological responses against C. trachomatis and it might be mediated by CXCL1 and CXCL9 but not CXCL10 and CXCL12. Based on these findings, these chemokines could be considered as future targets for immunotherapy to eradicate the $C$. trachomatis infection and its complications including infertility.

\section{Authors' Contributions}

All authors contributed extensively to the work presented in this paper.

\section{Funding/Support}

This project was supported by Rafsanjan University of Medical Sciences.

\section{References}

1. Keck C, Gerber-Schafer C, Clad A, Wilhelm C, Breckwoldt M. Seminal tract infections: impact on male fertility and treatment options. Hum Reprod Update. 1998;4(6):891-903.

2. Hamdad-Daoudi F, Petit J, Eb F. Assessment of Chlamydia trachomatis infection in asymptomatic male partners of infertile couples. J Med Microbiol. 2004;53(Pt 10):985-90.

3. Pawlowska A, Niemiec KT, Filipp E, El Midaoui A, Scholz A, Marianowska S, et al. Chlamydia trachomatis infection in pregnant women hospitalised in the Institute of Mother and Child in Warsaw, Poland. Med Wieku Rozwoj. 2005;9(1):21-6.

4. Dan M, Samra Z, Siegel YI, Korczak D, Lindner A. Isolation of Chlamydia trachomatis from prostatic tissue of patients undergoing transurethral prostatectomy. Infection. 1991;19(3):162-3.
5. Al-Mously N, Eley A. Interaction of Chlamydia trachomatis serovar E with male genital tract epithelium results in secretion of proinflammatory cytokines. J Med Microbiol. 2007;56(Pt 8):1025-32.

6. Aminzadeh F, Ghorashi Z, Nabati S, Ghasemshirazi M, Arababadi MK, Shamsizadeh A, et al. Differential expression of CXC chemokines CXCL10 and CXCL12 in term and pre-term neonates and their mothers. Am J Reprod Immunol. 2012;68(4):338-44.

7. Berlier W, Bourlet T, Levy R, Lucht F, Pozzetto B, Delezay O. Amount of seminal IL-1beta positively correlates to HIV-1 load in the semen of infected patients. J Clin Virol. 2006;36(3):204-7.

8. Hanada H, Ikeda-Dantsuji Y, Naito M, Nagayama A. Infection of human fibroblast-like synovial cells with Chlamydia trachomatis results in persistent infection and interleukin-6 production. Microb Pathog. 2003;34(2):57-63.

9. Derakhshan R, Arababadi MK, Ahmadi Z, Karimabad MN, Salehabadi VA, Abedinzadeh M, et al. Increased circulating levels of SDF-1 (CXCL12) in type 2 diabetic patients are correlated to disease state but are unrelated to polymorphism of the SDF-1beta gene in the Iranian population. Inflammation. 2012;35(3):900-4.

10. Zaja-Milatovic S, Richmond A. CXC chemokines and their receptors: a case for a significant biological role in cutaneous wound healing. Histol Histopathol. 2008;23(11):1399-407.

11. Mehrad B, Strieter RM. CXC Chemokine Signaling in Interstitial Lung Diseases. Intercellular Signaling in Development and Disease. Cell Signaling colec. 2011:441.

12. Hassanshahi G, Arababadi MK, Khoramdelazad H, Yaghini N, Zarandi ER. Assessment of CXCL12 (SDF-1alpha) polymorphisms and its serum level in posttransfusion occult HBV-infected patients in Southeastern Iran. Arch Med Res. 2010;41(5):338-42.

13. Hassanshahi G, Jafarzadeh A, Ghorashi Z, Zia Sheikholeslami N, Dickson AJ. Expression of IP-10 chemokine is regulated by proinflammatory cytokines in cultured hepatocytes. Iran J Allergy Asthma Immunol. 2007;6(3):115-21.

14. Liang Z, Brooks J, Willard M, Liang K, Yoon Y, Kang S, et al. CXCR4 CXCL12 axis promotes VEGF-mediated tumor angiogenesis through Akt signaling pathway. Biochem Biophys Res Commun. 2007;359(3):716-22.

15. Rottenberg ME, Gigliotti Rothfuchs AC, Gigliotti D, Svanholm C, Bandholtz L, Wigzell H. Role of innate and adaptive immunity in the outcome of primary infection with Chlamydia pneumoniae, as analyzed in genetically modified mice. J Immunol. 1999;162(5):2829-36.

16. WHO Organization . laboratory manual for the examination of human semen and sperm-cervical mucus interaction.: Cambridge university press; 1999.

17. Iwasaki A. Antiviral immune responses in the genital tract: clues for vaccines. Nat Rev Immunol. 2010;10(10):699-711.

18. Morrison RP, Caldwell HD. Immunity to murine chlamydial genital infection. Infect Immun. 2002;70(6):2741-51.

19. Mackern Oberti JP, Breser ML, Nunez N, Maccioni M, Rodriguez $\mathrm{N}$, Wantia N, et al. Chemokine response induced by Chlamydia trachomatis in prostate derived CD45+ and CD45- cells. Reproduction. 2011;142(3):427-37.

20. Raman D, Sobolik-Delmaire T, Richmond A. Chemokines in health and disease. Exp Cell Res. 2011;317(5):575-89.

21. Trumstedt C, Eriksson E, Lundberg AM, Yang TB, Yan ZQ, Wigzell $\mathrm{H}$, et al. Role of IRAK4 and IRF3 in the control of intracellular infection with Chlamydia pneumoniae. J Leukoc Biol. 2007;81(6):1591-8.

22. Royuela M, de Miguel MP, Ruiz A, Fraile B, Arenas MI, Romo E, et al. Interferon-gamma and its functional receptors overexpression in benign prostatic hyperplasia and prostatic carcinoma: parallelism with c-myc and p53 expression. Eur Cytokine Netw. 2000;11(1):119-27.

23. Hook CE, Matyszak MK, Gaston JS. Infection of epithelial and dendritic cells by Chlamydia trachomatis results in IL-18 and IL-12 production, leading to interferon-gamma production by human natural killer cells. FEMS Immunol Med Microbiol. 2005;45(2):113-20. 